\title{
Effects of rapid thermal annealing on the structural and local atomic properties of ZnO: Ge nanocomposite thin films
}

Cite as: J. Appl. Phys. 117, 105303 (2015); https://doi.org/10.1063/1.4914522

Submitted: 31 December 2014 . Accepted: 28 February 2015. Published Online: 10 March 2015

Abdullah Ceylan, Abdul K. Rumaiz, Deniz Caliskan, Sadan Ozcan, Ekmel Ozbay, and J. C. Woicik
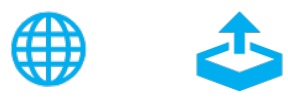

View Online

\section{ARTICLES YOU MAY BE INTERESTED IN}

Investigations of rapid thermal annealing induced structural evolution of $\mathrm{ZnO}$ : Ge nanocomposite thin films via GISAXS

Journal of Applied Physics 119, 215308 (2016); https://doi.org/10.1063/1.4953352

Diffusion of n-type dopants in germanium

Applied Physics Reviews 1, 011301 (2014); https://doi.org/10.1063/1.4838215

A comprehensive review of $\mathrm{ZnO}$ materials and devices

Journal of Applied Physics 98, 041301 (2005); https://doi.org/10.1063/1.1992666

Journal of Applied Physics
SPECIAL TOPIC:

Antiferromagnetic Spintronics 


\title{
Effects of rapid thermal annealing on the structural and local atomic properties of $\mathrm{ZnO}$ : Ge nanocomposite thin films
}

\author{
Abdullah Ceylan, ${ }^{1, a)}$ Abdul K. Rumaiz, ${ }^{2}$ Deniz Caliskan, ${ }^{3}$ Sadan Ozcan, ${ }^{1}$ Ekmel Ozbay, ${ }^{3,4,5}$ \\ and J. C. Woicik ${ }^{6}$ \\ ${ }^{1}$ SNTG Laboratory, Department of Physics Engineering, Hacettepe University, 06800 Ankara, Turkey \\ ${ }^{2}$ National Synchrotron Light Source, Brookhaven National Laboratory, Upton, New York 11973, USA \\ ${ }^{3}$ Nanotechnology Research Center, Bilkent University, 06800 Ankara, Turkey \\ ${ }^{4}$ Department of Physics, Bilkent University, 06800 Ankara, Turkey \\ ${ }^{5}$ Department of Electrical and Electronics Engineering, Bilkent University, 06800 Ankara, Turkey \\ ${ }^{6}$ National Institute of Standards and Technology, Gaithersburg, Maryland 20899, USA
}

(Received 31 December 2014; accepted 28 February 2015; published online 10 March 2015)

\begin{abstract}
We have investigated the structural and local atomic properties of Ge nanocrystals (Ge-ncs) embedded $\mathrm{ZnO}$ ( $\mathrm{ZnO}: \mathrm{Ge}$ ) thin films. The films were deposited by sequential sputtering of $\mathrm{ZnO}$ and Ge thin film layers on z-cut quartz substrates followed by an ex-situ rapid thermal annealing (RTA) at $600{ }^{\circ} \mathrm{C}$ for 30,60 , and $90 \mathrm{~s}$ under forming gas atmosphere. Effects of RTA time on the evolution of Ge-ncs were investigated by x-ray diffraction (XRD), scanning electron microscopy (SEM), hard x-ray photoelectron spectroscopy (HAXPES), and extended x-ray absorption fine structure (EXAFS). XRD patterns have clearly shown that fcc diamond phase Ge-ncs of sizes ranging between 18 and $27 \mathrm{~nm}$ are formed upon RTA and no Ge-oxide peak has been detected. However, cross-section SEM images have clearly revealed that after RTA process, Ge layers form varying size nanoclusters composed of Ge-ncs regions. EXAFS performed at the Ge K-edge to probe the local atomic structure of the Ge-ncs has revealed that as prepared $\mathrm{ZnO}$ :Ge possesses Geoxide but subsequent RTA leads to crystalline Ge structure without the oxide layer. In order to study the occupied electronic structure, HAXPES has been utilized. The peak separation between the $\mathrm{Zn} 2 p$ and Ge $3 d$ shows no significant change due to RTA. This implies little change in the valence band offset due to RTA. (C) 2015 AIP Publishing LLC. [http://dx.doi.org/10.1063/1.4914522]
\end{abstract}

\section{INTRODUCTION}

Within the quest for alternative high efficiency solar cells, intermediate bandgap solar cells have been proposed and extensively studied. ${ }^{1,2}$ The energy level of a confined state in a quantum dot (QD) in essence can act as an intermediate band. Thus, semiconducting nanoparticles embedded in a wide bandgap semiconducting host material establish an intermediate band making it possible for valence to intermediate band and subsequent intermediate to conduction band transitions thereby giving rise to enhanced spectral response. This approach is suggested to overcome the thermodynamic limit known as Shockley-Queisser (SQ) limit. ${ }^{3}$ Theoretical studies present conversion efficiency values between $46 \%$ and $86 \%$ depending on the assumptions of the models. ${ }^{4,5}$ However, these numbers are yet to be reached by experimental studies.

Significant efforts in QDs are directed on silicon (Si) and germanium $(\mathrm{Ge})$ nanocrystals embedded in a wide band gap oxide. ${ }^{6-9}$ The indirect band gap of both $\mathrm{Si}$ and Ge leads to a longer lifetime for electron-hole pairs as compared to direct bandgap materials. ${ }^{10}$ Although several authors have presented promising results on $\mathrm{Si}$ nanocrystals, Ge with a larger Bohr radius of about $24 \mathrm{~nm}$ as compared to that of $\mathrm{Si}$ (about $5 \mathrm{~nm}$ ) is expected to have a pronounced quantum

\footnotetext{
a) Author to whom correspondence should be addressed. Electronic mail: aceylanabd@yahoo.com Tel.: +90 31229772 29. Fax: +90 3122993037
}

confinement effect. ${ }^{11,12}$ Furthermore, the energy difference between indirect and direct band transitions for Ge being about $0.12 \mathrm{eV}$ making it even more attractive for the purpose of changing the electronic structure around the band edge. Early studies on Ge nanocrystals (Ge-ncs) embedded host oxide thin films demonstrate significant change on the optical properties and observed changes are usually attributed to quantum confinement of electron hole pairs in Ge-ncs. ${ }^{13-16} \mathrm{It}$ is imperative to understand the structural nature of Ge-ncs as well as the nature of the interface between the Ge-ncs and oxide matrix so as to interpret the optical properties. The thrust of current literature is centered on Ge-ncs embedded in $\mathrm{SiO}_{2}$ due to the ease of compatibility in standard $\mathrm{Si}$ based semiconductor processing. However, $\mathrm{ZnO}$ appears to be a better candidate as a wide bandgap structure for embedding quantum confined $\mathrm{Ge}$ nanoparticles. $\mathrm{ZnO}$ has various advantageous features like larger exciton binding energy $(\sim 60 \mathrm{meV})$ as compared to other wide band gap materials (such as $\mathrm{GaN}$ and $\mathrm{ZnSe}$ ) and high resistance to photo corrosion. Furthermore, the electron affinities of $\mathrm{ZnO}$ and $\mathrm{Ge}$ are very close to each other such that light generated charge carriers are not affected by a confining potential. In addition, the high-energy difference $(2.7 \mathrm{eV})$ between the valance bands of $\mathrm{Ge}$ and $\mathrm{ZnO}$ in the heterojunctions would give rise to a low leakage current.

In the literature, there is limited number of studies on Ge nanoparticles embedded $\mathrm{ZnO}$ thin film structures. ${ }^{13,17-19}$ In almost all of the referenced works, similar route of 
synthesis has been followed, that is, sequential sputtering of $\mathrm{Ge}$ and $\mathrm{ZnO}$ layers followed by a conventional furnace annealing. However, in this work, we have explicitly utilized rapid thermal annealing (RTA), as it is known to be an effective thermal process for the crystallization $\mathrm{Ge}$ nanoparticles. $^{20}$ Although present literature provides valuable information on the crystal structure and optical properties, there has not been any work presenting detailed results on the evolution of Ge-ncs via RTA process. Therefore in this work, with the aim of gaining insight on the variation of local atomic structure of Ge-ncs, we have performed ex-situ structural and local electronic studies using $x$-ray diffraction (XRD), scanning electron microscopy (SEM), extended $\mathrm{X}$-ray absorption fine structure (EXAFS), and hard x-ray photoelectron spectroscopy (HAXPES) measurements, respectively.

\section{EXPERIMENTAL DETAILS}

\section{A. Sample preparation}

Multilayered thin film samples were deposited by sequential sputtering of 2 in. $\mathrm{ZnO}$ and Ge targets with $99.99 \%$ purity. $100 \mathrm{~W}$ r.f. and d.c. sputter powers were applied to $\mathrm{ZnO}$ and $\mathrm{Ge}$ targets, respectively. $45 \mathrm{mT}$ Torr argon was employed as the sputtering gas. Four z-cut quartz substrates were cleaned in an ultrasonicator using in the order of acetone, alcohol, and distilled water prior to loading on a rotating substrate holder. The sample holder was kept at ambient temperature during the deposition process. Prior to the film deposition, vacuum chamber was pumped down to below $10^{-6}$ Torr and system was three times flushed with 500 mTorr Ar so as to reduce partial pressures of residual gas contents. Five layers of Ge were sequentially stacked between six layers of $\mathrm{ZnO}$. By adjusting the deposition times, $\mathrm{ZnO}$ and $\mathrm{Ge}$ layer thicknesses were adjusted to be about 90 and $20 \mathrm{~nm}$, respectively. Top ZnO layer was deposited for a longer time in order to avoid out diffusion of $\mathrm{Ge}$ atoms. The films were subsequently annealed in a commercial rapid thermal annealing set up under forming gas environment $\left(5 \% \mathrm{H}+95 \% \mathrm{~N}_{2}\right)$ at $600{ }^{\circ} \mathrm{C}$ for annealing times of 30,60 , and $90 \mathrm{~s}$. To avoid variation during deposition, the samples reported in this study were simultaneously grown.

\section{B. Characterization}

XRD studies were conducted by using a commercial diffractometer using $\mathrm{CuK}_{\alpha}$ radiation at a scanning step of $0.02^{\circ}$ in the $2 \theta$ range from $20^{\circ}$ to $65^{\circ}$. SEM measurements were conducted to obtain surface and cross section images of the samples. In order for the cross section, focused ion beam system has been utilized and images were taken in back scattering mode so as to obtain contrast due to atomic mass differences. HAXPES measurements were performed at the National Institute of Standards and Technology (NIST) beamline X24A at the National Synchrotron Light Source (NSLS), Brookhaven National Laboratory. The double crystal monochromator was operated using Si (111) crystals and high-resolution photoelectron spectra were collected using a hemispherical analyzer. EXAFS data collection was

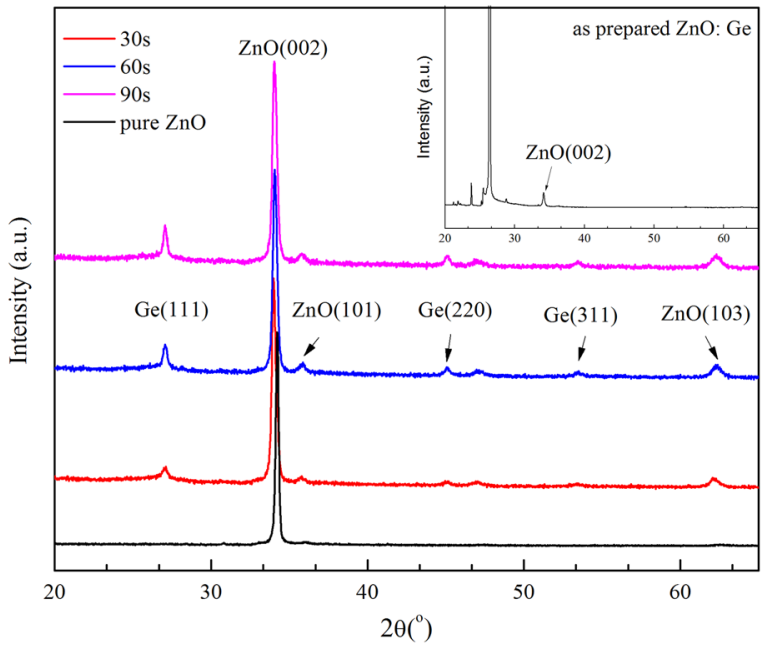

FIG. 1. XRD patterns of $\mathrm{ZnO}$ : Ge and pure $\mathrm{ZnO}$ thin films. Inset: XRD pattern of the as prepared $\mathrm{ZnO}$ : Ge sample.

performed using the fluorescence mode at beamline NIST X23-A2 at NSLS. To avoid the Bragg peak contamination due to the substrate, the sample was spun around its surface normal and the experiment was performed in grazing incidence. This procedure has produced extremely accurate EXAFS for single crystal materials. ${ }^{21,22}$ EXAFS data were analyzed using the IFEFFIT package. ${ }^{23}$

\section{RESULTS AND DISCUSSIONS}

XRD patterns of the as prepared and RTAed $\mathrm{ZnO}: \mathrm{Ge}$ samples at $600{ }^{\circ} \mathrm{C}$ for $30 \mathrm{~s}, 60 \mathrm{~s}$, and $90 \mathrm{~s}$ are presented in Figure 1. XRD patterns of $\mathrm{ZnO}$ and $\mathrm{Ge}$ have been investigated based on JCPDS\# 75-0576 and JCPDS\# 04-0545, respectively. The structural parameters extracted from the patterns are summarized in Table I. XRD pattern of the as prepared $\mathrm{ZnO}: \mathrm{Ge}$ in the inset of the figure shows no sign of $\mathrm{Ge}$ and reveals that $\mathrm{ZnO}$ has a weak crystalline structure. The diffraction peaks of z-cut quartz substrate dominate the overall pattern. However, RTA process leads to a welldefined c-axis oriented $\mathrm{ZnO}$ crystal structure as shown in Figure 1. It has been understood by using Scherrer calculations that while pure $\mathrm{ZnO}$ structure possesses $36 \mathrm{~nm} \mathrm{ZnO}$ crystallites, Ge-ncs embedded $\mathrm{ZnO}$ structure, however, acquires smaller $(\sim 24 \mathrm{~nm}) \mathrm{ZnO}$ crystallites. Moreover, we notice that Ge-ncs inclusion leads to $0.7 \%$ increase in $a, b$ parameters, while $c$ does not significantly change compared to pure $\mathrm{ZnO}$ sample. Although XRD pattern of the as

TABLE I. Crystallite size and lattice constants extracted from XRD patterns of the samples.

\begin{tabular}{|c|c|c|c|c|c|}
\hline \multirow{2}{*}{$\begin{array}{l}\text { RTA } \\
\text { time } \\
\text { (s) }\end{array}$} & \multirow{2}{*}{$\begin{array}{l}\mathrm{Ge} \\
\text { crystallite } \\
\text { size }(\mathrm{nm})\end{array}$} & \multirow{2}{*}{$\begin{array}{c}\text { Ge lattice } \\
\text { constant } \\
(\AA)\end{array}$} & \multirow{2}{*}{$\begin{array}{c}\mathrm{ZnO} \\
\text { crystallite } \\
\text { size }(\mathrm{nm})\end{array}$} & \multicolumn{2}{|c|}{ ZnO lattice constants $(\AA)$} \\
\hline & & & & $a=b$ & $c$ \\
\hline 30 & 18.2 & 5.706 & 23.91 & 3.291 & 5.263 \\
\hline 60 & 24.7 & 5.702 & 25.18 & 3.289 & 5.259 \\
\hline 90 & 26.7 & 5.702 & 23.58 & 3.290 & 5.262 \\
\hline $\begin{array}{l}\text { Pure } \\
\mathrm{ZnO}\end{array}$ & $\ldots$ & $\ldots$ & 35.27 & 3.267 & 5.256 \\
\hline
\end{tabular}




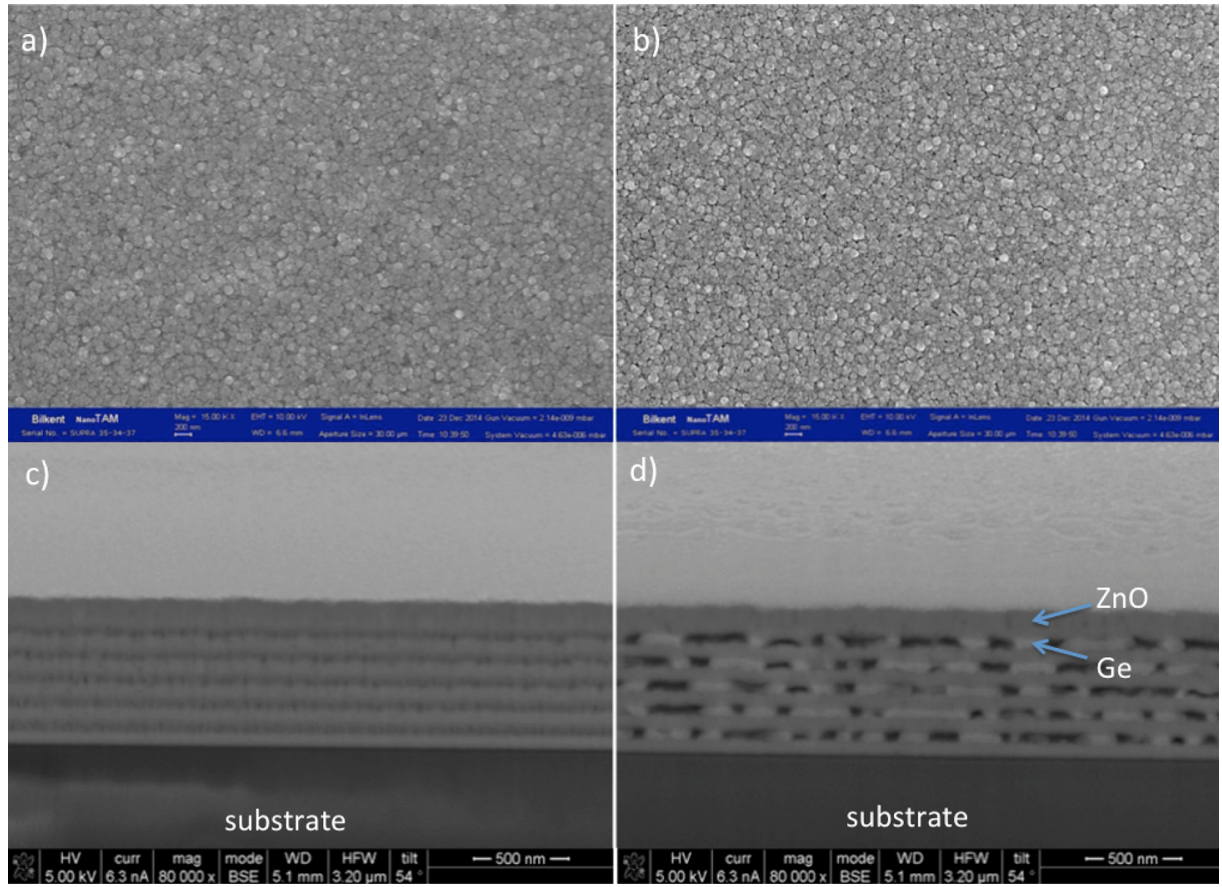

FIG. 2. Surface and cross-section SEM images of as prepared (a) and (c) and $60 \mathrm{~s}$ RTA processed sample (b) and (d). prepared sample does not reveal any Ge diffraction peak, it is clear from the patterns that RTA process leads to the formation of Ge-ncs with fcc diamond lattice. It is readily seen that increasing RTA time from $30 \mathrm{~s}$ to $60 \mathrm{~s}$ gives rise to $26.3 \%$ increase in the Ge crystallite size while the 90 s RTA changes the crystallite size by only $7.5 \%$ reaching to $26.7 \mathrm{~nm}$. This is about the expected crystallite size limit from $\mathrm{a} \sim 20 \mathrm{~nm}$ Ge layer. Beyond this, we expect very little change in the crystallite size with increasing the RTA time.

In Figure 2, surface (a) and (b) and cross-sectional (c) and (d) SEM images of $60 \mathrm{~s}$ RTA processed ZnO:Ge thin films are presented. $\mathrm{ZnO}$ surface (Figs. 2(a) and 2(b)) has a granular polycrystalline structure and it appears that RTA process does not significantly affect the surface structure. Cross-section SEM images of the as prepared (Fig. 2(c)) and 60 s RTA processed (Fig. 2(d)) samples clearly demonstrate that RTA process leads to formation of Ge nanoclusters separated between $\mathrm{ZnO}$ layers each with an approximate layer thickness of $90 \mathrm{~nm}$. Notice that the atomic mass difference between $\mathrm{Zn}$ and $\mathrm{Ge}$ atoms reflects itself on the images as $\mathrm{Ge}$ regions being lighter as opposed to dark $\mathrm{ZnO}$ regions. Therefore, combining with XRD results, we understand that upon RTA process, Ge layers form varying size nanoclusters composed of small crystallites whose size extracted from the XRD patterns.

XRD patterns of all the samples do not reveal a detectable Ge-oxide formation, however previous studies show that there has to be a thin oxide interface layer between $\mathrm{Ge}$ and $\mathrm{ZnO},{ }^{16}$ which usually tends to be amorphous. Therefore with the aim of better understanding the chemical and electronic structure, we have performed HAXPES on the $\mathrm{ZnO}$ :Ge samples. The higher energy afforded by HAXPES enables us to probe deeper into the samples. Figure 3 shows the characteristic $\mathrm{Zn} 2 p$ core line for the as prepared and the RTAed samples. To further analyze the oxidation of $\mathrm{Ge}$, we plotted the Ge $3 d$ spectra of all the samples in Figure 3. For the ease of comparison, we have set the peak position of elemental $\mathrm{Zn}$ $2 p$ to be $0 \mathrm{eV}$. One can see a clear evolution of the Ge $3 d$ spin doublet as a function of RTA time. Additionally, an extra peak about $3 \mathrm{eV}$ from the Ge $3 d$ spin doublet is readily seen. This corresponds to the $\mathrm{GeO}_{\mathrm{x}}$ peak and is below the reported value for $\mathrm{GeO}_{2} \cdot{ }^{24}$ The valence band offset of a semiconductor heterojunctions can be computed by comparing the core and the valence band of a reference semiconductor and by measuring the core lines from the actual heterojunctions using the well known Kraut's method. ${ }^{25}$ In other words, the change in valence band offset between $\mathrm{Ge}$ and $\mathrm{ZnO}$ is evident from the peak separation between $\mathrm{Zn} 2 p$ and Ge $3 d$ core lines. From Figure 4 , we see little change in the peak separation between $\mathrm{Zn} 2 p$ and Ge $3 d$ for the as prepared, and RTAed samples indicate that changes in the valence band offset between $\mathrm{Ge}$ and $\mathrm{Zn}$ resulting from particle size change are negligible. Although HAXPES does show a

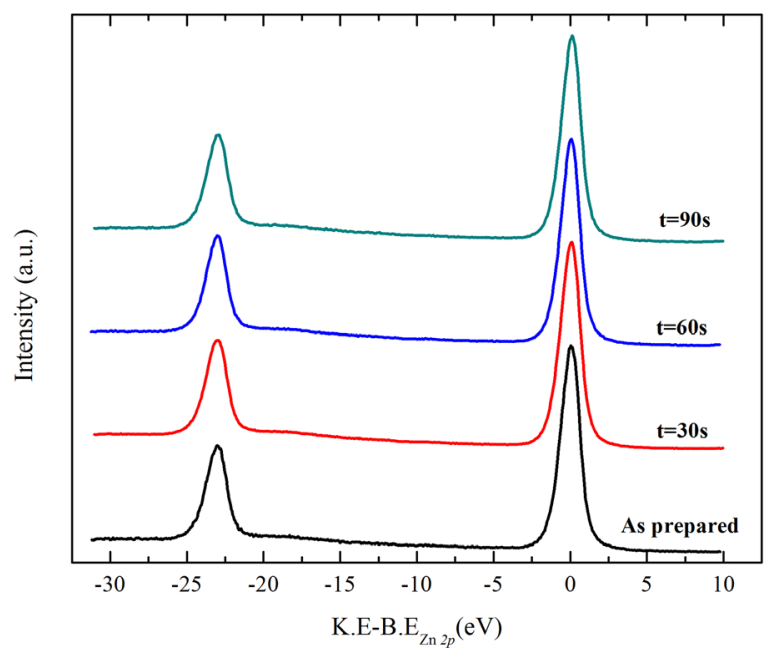

FIG. 3. Zn $2 p$ collected at $\mathrm{h} \nu=2150 \mathrm{eV}$ for the as prepared and RTA processed samples. The plot is referenced with respect to the $\mathrm{Zn} 2 \mathrm{p}_{3 / 2}$. 


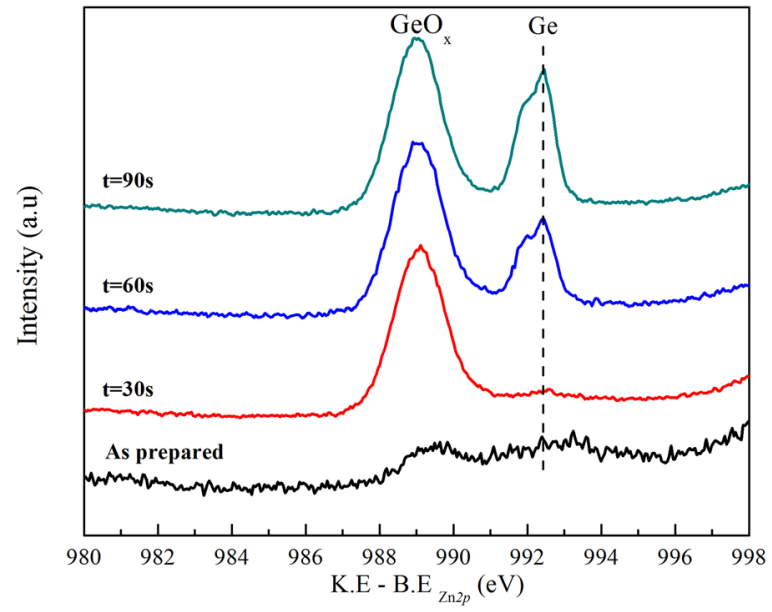

FIG. 4. Ge $3 d$ collected at $\mathrm{h} \nu=2150 \mathrm{eV}$ for the as prepared and RTA processed samples. The spectra are referenced with respect to $\mathrm{Zn} 2 \mathrm{p}_{3 / 2}$.

strong oxide shell growing with RTA it must be pointed out that with the conventional photoemission, we are still prone to probing the surface contribution. To probe the bulk structure, EXAFS measurements were performed on the Ge Kedge of the samples. The EXAFS data were analyzed using the IFEFFIT package. The AUTOBK code $^{26}$ was used to normalize the absorption coefficient $\mu(k)$ by the absorption edge step and separate the EXAFS $\chi(k)$ as

$$
\chi(k)=\frac{\mu(k)-\mu_{0}(k)}{\Delta \mu_{0}(k)},
$$

where $k$ is the photoelectron wave number. Fourier transformed magnitude of the Ge $\mathrm{K}$ edge data for the samples and a reference Ge sample are shown in Figure 5. The data have been analyzed with identical parameters and are plotted together for comparison. Visual inspection of the plot shows the magnitude of Fourier transform systematically increasing with annealing time. Furthermore, the appearance of the second shell contribution is evident from the annealed samples. These are indication of crystallization of the amorphous as prepared sample. Closer inspection of the EXAFS spectra

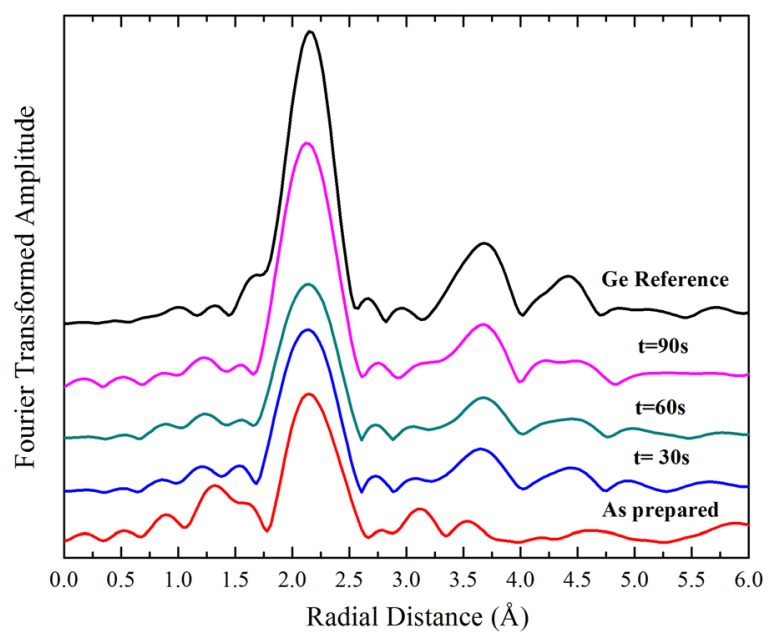

FIG. 5. Fourier transform magnitudes of Ge EXAFS as a function of annealing time. Ge reference data are included for comparison. shows a dominant peak close to $2.1 \AA$. This peak corresponds to the Ge-Ge first shell in the system. For the as prepared sample, in addition to the dominant peak, we see a weak peak at $1.4 \AA$. This peak corresponds to Ge-O first shell in the system. This feature is absent in the RTA processed samples. Thus upon annealing, concomitant with the crystallization of $\mathrm{Ge}$ is also the gettering of oxygen from the Ge by the $\mathrm{ZnO}$ host. Lysaght et al. have reported similar results for $\mathrm{HfO}_{2}$ grown on Ge substrates. ${ }^{27}$ Within the sensitivity afforded by EXAFS measurements, we can conclude that although as prepared sample does tend to have Ge-oxide, subsequent RTA gives rise to formation of Ge-ncs with well defined crystalline structure. Combining with structural characterization results of $\mathrm{ZnO}: \mathrm{Ge}$ thin film samples, we can conclude that $\mathrm{Ge}$ is initially in an amorphous state with an amorphous Ge-oxide shell. Subsequent RTA at $600^{\circ} \mathrm{C}$ initiates evolution of larger Ge crystallites while decreasing the Ge-oxide shell. EXAFS measurement clearly shows the crystallization of Ge and simultaneous gettering of oxygen by $\mathrm{ZnO}$ lattice.

\section{CONCLUSIONS}

RTA promoted evolution of Ge-ncs in $\mathrm{ZnO}$ host has been investigated. We have synthesized fcc diamond phase Ge-ncs with crystallite sizes ranging between 18 and $27 \mathrm{~nm}$ by sequential sputtering of $\mathrm{Ge}$ and $\mathrm{ZnO}$ targets followed by RTA at $600^{\circ} \mathrm{C}$. Detailed structural and local atomic characterization showed that RTA process produces Ge-ncs in the form of varying size nanoclusters between the $\mathrm{ZnO}$ thin film layers, and amorphous Ge layers with very thin Ge-oxide layers of the as prepared samples can be turned into well defined Ge-ncs without Ge-oxide formation upon RTA process implemented under certain conditions. This is in fact very important information as to the formation of confining potential for light generated charge carriers. Although, previous studies on the optical properties have shown significant difference with particle size of Ge-ncs, HAXPES measurements show no change in the occupied electronic structure of the $\mathrm{ZnO}: \mathrm{Ge}$ thin film system.

\section{ACKNOWLEDGMENTS}

This work was supported by The Scientific and Technical Research Council of Turkey (TUBITAK) with the Grant No. 109T722. The work was performed in National Synchrotron Light Source at Brookhaven National Laboratory supported by U.S. Department of Energy, Office of Science, Office of Basic Energy Sciences, under Contract No. DE-AC02-98CH10886.

\footnotetext{
${ }^{1}$ A. Luque and A. Marti, "Increasing the efficiency of ideal solar cells by photon induced transitions at intermediate levels," Phys. Rev. Lett. 78, 5014 (1997).

${ }^{2}$ A. Marti, E. Antolin, C. R. Stanley, C. D. Farmer, N. Lopez, P. Diaz, E. Canovas, P. G. Linares, and A. Luque, Phys. Rev. Lett. 97, 247701 (2006). ${ }^{3}$ W. Shockley and H. Queisser, J. Appl. Phys. 32, 510 (1961).

${ }^{4}$ A. Marti, L. Cuadra, and A. Luque, "Design constraints of the quantum dot intermediate solar cell," Physica E 14, 150 (2002).

${ }^{5}$ A. de Vos, "Detailed balance limit of the efficiency of tandem solar cells," J. Phys. D 13, 839 (1980).
} 
${ }^{6}$ S. Cosentino, S. Mirabella1, M. Miritello, G. Nicotra, R. Lo Savio, F. Simone, C. Spinella, and A. Terrasi, "The role of the surfaces in the photon absorption in Ge nanoclusters embedded in silica," Nanoscale Res. Lett. 6, 135 (2011).

${ }^{7}$ C. Uhrenfeldt, J. Chevallier, A. N. Larsen, and B. B. Nielsen, "Nearinfrared-ultraviolet absorption cross-sections for Ge nanocrystals in $\mathrm{SiO} 2$ thin films: Effects of shape and layer structure," J. Appl. Phys. 109, 094314 (2011).

${ }^{8}$ D. Dawei, I. P. Wurfl, A. Gentle, D. H. Kim, X. Hao, L. Shi, G. Conibeer, and M. A. Green, "Impacts of post metallization process on the electrical and photovoltaic properties of Si quantum dot solar cells," Nanoscale Res. Lett. 5, 1762 (2010).

${ }^{9}$ M. Ficcadenti et al., "Si quantum dots for solar cell fabrication," Mater. Sci. Eng., B 159-160, 66 (2009).

${ }^{10}$ D. Timmerman, I. Izeddin, P. Stallinga, I. N. Yassievich, and T. Gregorkiewicz, "Space-separated quantum cutting with silicon nanocrystals for photovoltaic applications," Nat. Photonics 2, 105 (2008).

${ }^{11}$ Y. Maeda, N. Tsukamoto, Y. Yazawa, and Y. Masumoto, "Visible photoluminescence of Ge microcrystals embedded in $\mathrm{SiO} 2$ glassy matrices," Appl. Phys. Lett. 59, 3168 (1991).

${ }^{12}$ A. G. Cullis, L. T. Canham, and P. D. Calcott, "The structural and luminescence properties of porous silicon,” J. Appl. Phys. 82, 909 (1997).

${ }^{13}$ U. Pal, G. C. Segura, and O. Z. Corona, "Preparation of Ge/ZnO nanocomposites by radio frequency alternate sputtering," Sol. Energy Mater. Sol. Cells 76, 305 (2003).

${ }^{14}$ S. Foss, T. G. Finstad, A. Dana, and A. Aydinli, "Growth of Ge nanoparticles on $\mathrm{SiO}_{2} / \mathrm{Si}$ interfaces during annealing of plasma enhanced chemical vapor deposited thin films," Thin Solid Films 515, 6381 (2007).

${ }^{15}$ F. Gao, M. A. Green, G. Conibeer, E. C. Cho, Y. Huang, I. P. Wurfl, and C. Flynn, "Fabrication of multilayered Ge nanocrystals by magnetron sputtering and annealing," Nanotechnology 19, 455611 (2008).
${ }^{16}$ S. D. Singh, R. S. Ajimsha, V. Sahu, R. Kumar, P. Misra, D. M. Phase, S. M. Oak, L. M. Kukreja, T. Ganguli, and S. K. Deb, "Band alignment and interfacial structure of $\mathrm{ZnO} / \mathrm{Ge}$ heterojunction investigated by photoelectron spectroscopy," Appl. Phys. Lett. 101, 212109 (2012).

${ }^{17} \mathrm{U}$. Pal and J. G. Serrano, "Raman and infrared spectroscopy of Ge nanoparticles embedded in ZnO matrix,” Appl. Surf. Sci. 246, 23 (2005).

${ }^{18}$ G. H. Shih, C. G. Allen, and B. G. Potter, Jr., Nanotechnology 23, 075203 (2012).

${ }^{19}$ A. Ceylan, J. M. Ali, and S. Ozcan, "Synthesis of $\mathrm{ZnO}$ : Ge nanocomposite thin films by plasma gas condensation," Mater. Sci. Semicond. Process. 16, 424 (2013).

${ }^{20}$ A. F. Abd Rahim, M. R. Hashim, N. K. Ali, M. Rusop, M. D. Johan Ooi, and M. Z. M. Yusoff, "Self-assembled Ge islands and nanocrystals by RF magnetron sputtering and rapid thermal processing: The role of annealing temperature," Appl. Surf. Sci. 275, 193 (2013).

${ }^{21}$ J. C. Woicik, J. A. Gupta, and S. P. Watkins, "Bond length strain in buried $\mathrm{Ga}_{1-\mathrm{x}} \mathrm{In}_{\mathrm{x}} \mathrm{As}$ thin-alloy films grown coherently on $\mathrm{InP}(001)$," Appl. Phys. Lett. 73, 1269 (1998).

${ }^{22}$ J. C. Woicik, "Random-cluster calculation of bond lengths in strainedsemiconductor alloys," Phys. Rev. B 57, 6266 (1998).

${ }^{23} \mathrm{M}$. Newville, "EXAFS analysis using FEFF and FEFFIT," J. Synchrotron Radiat. 8, 322 (2001).

${ }^{24}$ A. K. Rumaiz, J. C. Woicik, C. Wieland, Q. Xie, D. P. Siddons, G. H. Jaffari, and C. Detavernier, Appl. Phys. Lett. 101, 222110 (2012).

${ }^{25}$ E. A. Kraut, R. W. Grant, J. R. Waldrop, and S. P. Kowalczyk, Phys. Rev. Lett. 44, 1620 (1980).

${ }^{26}$ M. Newville, P. Livins, Y. Yacoby, J. J. Rehr, and E. A. Stern, Phys. Rev. B 47, 14126 (1993).

${ }^{27}$ P. S. Lysaght, J. Barnett, G. I. Bersuker, J. C. Woicik, D. A. Fischer, B. Foran, H. H. Tseng, and R. Jammy, "Chemical analysis of $\mathrm{HfO}_{2} / \mathrm{Si}$ (100) film system exposed to $\mathrm{NH}_{3}$ thermal processing," J. Appl. Phys. 101, 024105 (2007). 